\title{
Éthnica. Revista de Antropología y su significación histórica para la Antropología en España ${ }^{1}$
}

\author{
Luis Calvo Calvo \\ Institución Milá y Fontanals. CSIC. Barcelona
}

\section{RESUMEN}

El artículo presenta lo que supuso la revista Éthnica, fundada por Claudio Esteva Fabregat en el Centro de Etnología Peninsular del CSIC de Barcelona a principios de la década de 1970. Éthnica, que finalizó su andadura en 1985, se convirtió, como tal, en la primera revista de antropología cultural y social española, dejando un legado que llega hasta nuestros días por la amplitud y el rigor de los temas tratados a lo largo de los veinte volúmenes que se publicaron.

Palabras clave: Etnología, Antropología, Cataluña, España, Claudio Esteva Fabregat.

\section{SUMMARY}

The author discusses the scholarly accomplishment that Ethnica represented in Spanish anthropology. Founded in the early 1970s by Claudio Esteva Fabregat in the Centro de Etnología Peninsular of the CSIC in Barcelona, the journal soon became the most respected publication of social and cultural anthropology in Spain. Though ended in 1985, Ethnica left a valuable heritage of twenty volumes in which wide-ranging research interests appear combined with analytical rigor.

Key words: Ethnology, Anthropology, Catalonia, Spain, Claudio Esteva Fabregat.

\section{INTRODUCCIÓN}

La antropología española inició su auténtica institucionalización a raíz de la creación del Departamento de Antropología Cultural de la Universi-

\footnotetext{
${ }^{1}$ Una primera version de este artículo se publicó en 1996 con el título "Claudio Esteva Fabregat, Éthnica y la Antropología", en J. Prat y Á. Martínez (eds.), Ensayos de antropologia cultural. Homenaje a Claudio Esteva-Fabregat: 42-49. Barcelona: Ariel.

RDTP, LVII, 1 (2002): 71-82
} 
dad de Barcelona en 1971. Desde aquel entonces la disciplina se ha asentado en la universidad española y ha empezado a tener una proyección social notable.

Julio Caro Baroja, Carmelo Lisón Tolosana y Claudio Esteva Fabregat han sido las personas que, cada uno con su específica manera de entender la antropología, han fundamentando con sus investigaciones y con sus actuaciones buena parte de lo desarrollado en este período. Como partícipe de esta "tríada fundacional", Esteva ha jugado un papel ciertamente singular, por sus tareas favorables a la plena incorporación de la antropología al medio académico, por su destacado papel en la formación de antropólogos, por la creación de adecuados canales de difusión $\mathrm{y}$, finalmente, por sus aportaciones formales y conceptuales a la disciplina.

En este texto, quiero ahondar en la significación que tuvo Éthnica en un momento determinado de acceso e inicio de consolidación de la antropología en la universidad española.

Claudio Esteva Fabregat: PersPectivas teóRICas para ÉthNica. REVISTA DE ANTROPOLOGÍA

El denominado "giro" de la antropología (Buxó 1994; Delgado 1993) ha propiciado el desarrollo de la reflexividad del conocimiento antropológico que, a su vez, ha permitido que, más allá del análisis de los parámetros y paradigmas sobre los que se ha erigido la antropología contemporánea, otros objetos o temáticas puedan proporcionar altas cotas de información sobre el devenir y la construcción de la etnografia, reflexividad que "[...] ha hecho que se haya analizado, de forma mucho más minuciosa, [cómo] los antropólogos han construido su ciencia. [De esta formal tomando como objeto de estudio el autor y su medio, los antropólogos y los historiadores de la antropología han empezado a reinterpretar y a revaluar las etnografías escritas por sus antepasados" (Geary 1990: 289-290).

Este planteamiento permite acercarse más y mejor a cómo se gestó Éthnica, para lo cual es necesario recurrir, aunque sea de forma breve, a la trayectoria de Esteva Fabregat y al contexto académico e intelectual en el que nació dicha publicación. Así, hay que tener en cuenta que, a finales de la década de 1970, se tuvo que realizar un giro de ciento ochenta grados con vistas a delimitar el campo de actuación de la antropología española, lo que permitió, entre otras cosas, dotar de una organización adecuada para la consolidación de la materia en el medio académico y en la sociedad, así como realizar un auténtico tour de force con vistas a 


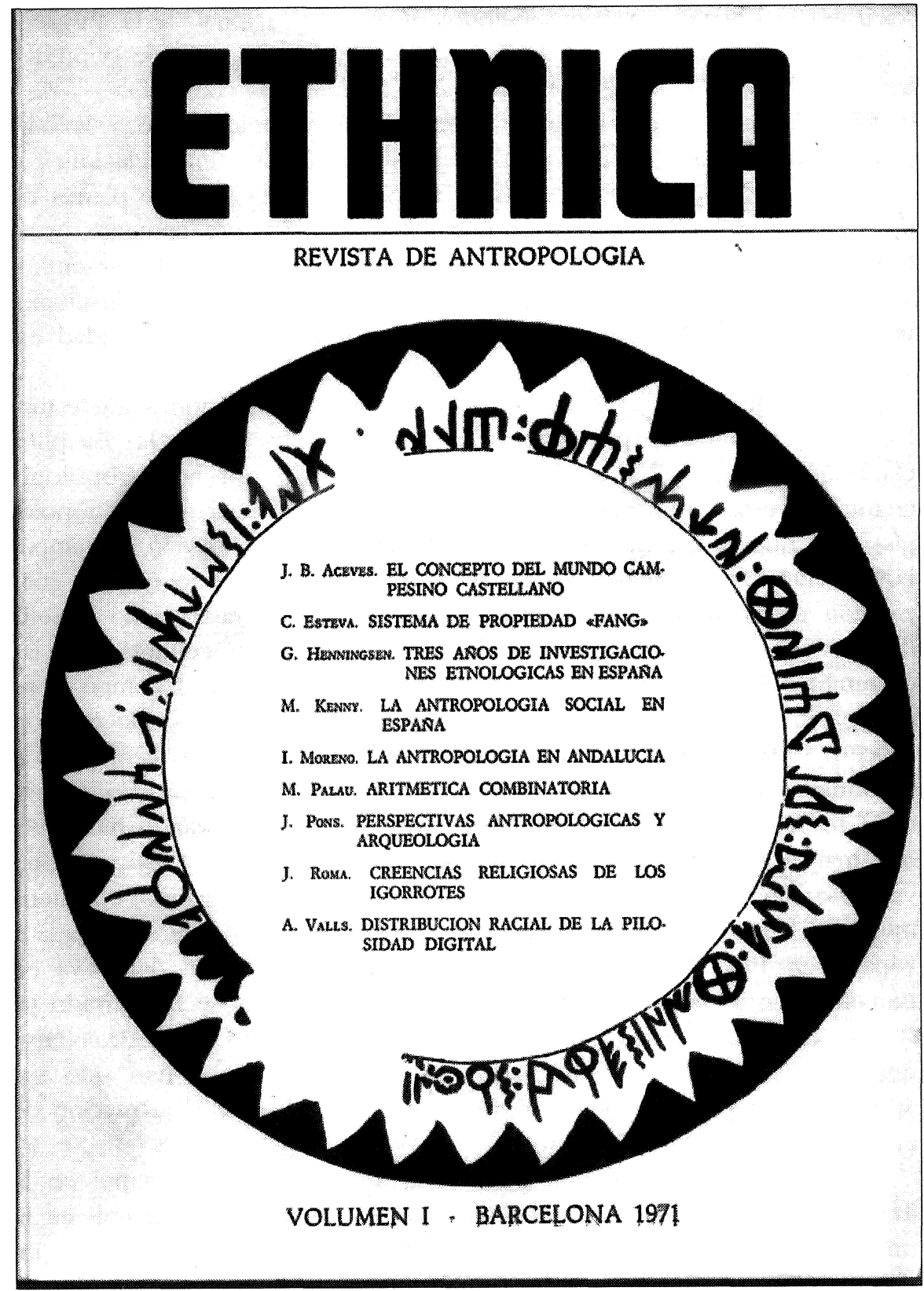


proporcionarle suficientes instrumentos teóricos y metodológicos que le permitiesen ser reconocida como una auténtica disciplina académica. Pero, ¿cuál era el horizonte teórico, metodológico e institucional de la antropología española en 1971? Básicamente, era: 1. la dependencia de la prehistoria y de la arqueología; 2 . la utilización de métodos conjeturales y descriptivos; 3. el escaso trabajo de campo; 4. el autodidactismo y la baja profesionalización; 5. la ausencia de financiación para investigaciones u otros servicios (ediciones de revistas, libros...) y 6. la falta de planes de estudio (Esteva 1969; citado por Prat 1992: 21). En este contexto, ¿cuál fue la orientación por la que Esteva apostó? y ¿qué "actitud epistémica" adoptó con vistas a la consecución de sus fines, dirigidos a la institucionalización de la antropología como ciencia propia en la universidad española?

Esteva Fabregat, científico formado en diversas tradiciones intelectuales, se decantó desde un principio por una concepción de la disciplina claramente dirigida hacia el modelo propugnado por la antropología culturalista estadounidense de tradición boasiana; es decir, una antropología en la que lo orgánico y lo superorgánico, lo pretérito y lo contemporáneo, eran claves de bóveda de la tarea a realizar, por lo que su concepción de la antropología cultural pasó por considerarla como aquella "[...] ciencia que se ocupa del estudio comparado de las culturas y de las costumbres humanas en su evolución y adaptaciones en el tiempo y en el espacio [siendo] su objetivo el conocimiento de las leyes que rigen el proceso histórico de las formas culturales en sus variedades ecológicas y su transmisión y transformaciones" (Esteva 1993: 26). Este posicionamiento lo ha reafirmado en múltiples ocasiones, en especial cuando ha tenido que matizar las diferencias entre la antropología cultural y la social (Esteva 1994). De hecho, esta concepción de la disciplina condicionó, en buena medida, el trabajo científico e institucional que realizó desde su llegada a España en 1956 y, sobre todo, a partir de su instalación definitiva en Barcelona en 1968. A pesar de que en otras ocasiones se ha narrado ya su trayectoria científica (Aguirre 1986 y 1994; Calvo 1985 y 1993), pienso que todavía no se han explicitado suficientemente cuáles han sido sus aportaciones teóricas y metodológicas fundamentales a la construcción de la antropología cultural española.

En primer lugar, hay que destacar la relevancia de su papel en la introducción y la extensión del concepto de "antropología cultural" en la universidad española de los años sesenta y setenta; ello supuso una innovación de notable trascendencia, dado que hasta aquel momento la etnología estaba supeditada a otras disciplinas y su relación con ellas se basaba en la desigualdad. Privilegiar un concepto sobre otro -antropolo- 
gía cultural versus etnología ${ }^{2}$ - supuso, a principios de la década de 1970, un dilema importante. La creación del Departamento de Antropología Cultural de la Universidad de Barcelona en 1971 y, al año siguiente, de la Cátedra de Antropología Cultural, le llevó a apostar decididamente por el primer concepto, mas no debemos llamarnos a error: lo que intentó fue acotar y marcar diferencias con otras ciencias sociales y humanas. En este sentido, debe remarcarse la perspicacia que tuvo al ver la absoluta necesidad de establecer un nuevo estatuto entre la antropología y la prehistoria y la arqueología u otras materias, todo ello con vistas a potenciar la igualdad de discursos y conseguir un ámbito de trabajo propio, con metodologías y teorías propias. Asimismo, buscó situar a la etnología en el lugar que realmente le corresponde en el discurso y en la concepción contemporánea de la antropología cultural y social. De esta manera, Esteva no rechazó el concepto de etnología, sino que lo dotó de su dimensión real, es decir, ser un estadio más del trabajo antropológico. Así, se afianzó la idea del comparativismo como una de las marcas diferenciales de la nueva disciplina en la universidad española de aquellos años. Este posicionamiento teórico se plasmó en su propia trayectoria académica, en la que en sus primeros años en Madrid trató temas como los de "Etnología Andina" o los de "Etnología Azteca" y, con posterioridad, y ya en la Universidad de Barcelona, incorporó asignaturas, que todavía subsisten en la actualidad, como "Etnología de los Pueblos Primitivos" o "Etnologia dels Països Catalans".

Su segunda aportación destacada fue hacer hincapié, con su formación funcionalista, en conceptos como los de "proceso" o "conflicto social, conceptos que, en buena medida, hàn determinado la transformación de la antropología contemporánea. Hay que recordar que, en el panorama de la antropología internacional, el modelo estructuralfuncionalista se fue modificando, gracias a la adquisición de nuevos intereses por los procesos evolutivos y por la relación entre variación y estructura (Voget 1984: 3). Así, el aspecto procesal de la investigación comportó que la antropología fijase menos su atención en los individuos y en las ideas y más en los procesos, que son observados en base a la relación dialéctica entre objeto social y cultural. Así, Esteva Fabregat abandonó anteriores formas de analizar la sociedad española que más que

\footnotetext{
${ }^{2} \mathrm{Al}$ respecto, Esteva (1982: 16) escribió: «En gran manera, además, era indispensable propiciar el desarrollo de una concepción teórica inclusiva más amplia y la Antropología Cultural cumplía estos requisitos [...] siguiendo un desarrollo con precedentes en las tradiciones antropológicas de EE.UU. e Iberoamérica, tenía que designarse con el nombre de Antropología Culturalw.
} 
estudiar el problema de la sociedad se planteaban "problemas respecto a..." (Gómez 1990: 26). Tal postura hizo que Esteva se interesase por el estudio de la realidad, no desde una posición idealista, deductiva exclusivamente, sino desde la inducción y desde la perspectiva de la implicación directa, gracias a la observación participante, en el conocimiento de la estructura sociocultural. En este sentido, sus orientaciones y sus trabajos se opusieron a la lógica de la fenomenología decimonónica, especialmente la alemana, que a la hora de hacer "teoría de la sociedad" privilegió la aparición de "[...] un "yo", un "tú", un "él", un "nosotros" — sobre todo, un místico "nosotros" "[por encima de] "los sujetos concretos de la vida social (hombre, obrero, patrono, ciudadano, campesino, etcétera)" [lo que provocó que] "la sociedad se [convirtiese] en una especie de Arcadia, en donde los sujetos se han disfrazado de pronombres personales" (Gómez 1990: 34-35).

Incidir en estos aspectos supuso una significativa redefinición conceptual y formal, lo que propició, entre otras cosas, el dejar de considerar que los sistemas culturales y sociales eran autolimitados y determinados $\mathrm{y}$, por lo tanto, el reconocimiento de que en ellos incidían múltiples factores que los iban conformando. Así, la causalidad empezó a ser vista como un proceso de compleja naturaleza, resultado de la incidencia de diversas variables (Voget 1984: 10), tal como el propio Esteva había puesto de manifiesto en sus estudios en el Alto Aragón y en sus trabajos sobre antropología aplicada. Todo ello supuso, entre otras cosas, la ruptura de las viejas estrategias de análisis de comunidades cerradas, lo que significó, además, evidenciar que el mundo rural también debía ser observado en función del mundo urbano. Estos planteamientos situaron, en definitiva, la nueva disciplina en la confrontación y en la dialéctica entre el mundo preindustrial y el mundo contemporáneo, lo que la dotó de carácter específico y propio.

Estas aportaciones teóricas se vieron complementadas por las de carácter metodológico; en este sentido, la "ruptura" de las explicaciones de carácter totalizador a través de la introducción de variables como la "procesal" o la del "conflicto social", comportó que la atención se fijase sobre las variables situacionales. Ello persiguió poner al descubierto cómo los procesos estructurales se modifican dinámicamente según las circunstancias; así, variabilidad y contradicción se presentaron como elementos claves en las explicaciones. Esteva puso todo ello en práctica a través de metodologías concretas, como es el caso de la funcionalista o la de cultura y personalidad.

Unos y otros aspectos llevaron al desarrollo de nuevos campos de investigación como, por ejemplo, el de la antropología lingüística, el cual 
debe ser visto como un anhelo más por conocer las estructuras y los procesos cognitivos (Voget 1984: 10), o los referidos al cambio sociocultural, la etnicidad, la aculturación o el mestizaje.

Esta serie de aspectos fueron, como ya he anticipado, una novedad en el contexto antropológico e intelectual de los años setenta en España, en especial el de la etnicidad, si se tienen presentes las corrientes ideológicas predominantes del momento que, en gran medida, negaban todo lo referido a la etnicidad ya que se consideraba que el análisis debía basarse en otros parámetros (clase social, etc.).

\section{ETHNICA. REVISTA DE ANTROPOLOGÍA}

El conjunto de aspectos citados acrecientan la relevancia de Ethnica. Revista de Antropología. La publicación nació en 1971 (Alcañiz 1994) con el ansia de proporcionar un espacio para presentar una forma concreta de hacer y entender la antropología como ciencia, configuración que tuvo en el holismo y en la interdisciplinariedad aspectos centrales ${ }^{3}$. De esta forma, la publicación respondió claramente al nuevo enfoque epistémico que nuestro autor propició, lo que supuso una nueva forma de aproximarse a la comprensión de los fenómenos socioculturales, ya fuesen pretéritos o contemporáneos. Por otra parte, la gestación y el desarrollo de Ethnica están íntimamente relacionados con la consolidación de la actividad científica y formativa que Esteva desarrolló en el Centro de Etnología Peninsular (a partir de 1978 se le añadió, "e Hispanoamericana") del Consejo Superior de Investigaciones Científicas en Barcelona.

Aunque Esteva proyectó su visión de la disciplina a través de la revista, lo que se reflejó, entre otras cosas, en un destacado peso de los temas americanistas, Ethnica se convirtió en un pilar para la institucionalización de la materia en unos momentos en los que la juventud de la disciplina hacía peligrar su definitiva instalación.

En un análisis más detallado de la publicación, el primer rasgo que llama la atención es el manifiesto deseo por integrar saberes, corrientes, tendencias, personajes, enfoques y puntos de vista. Hay que destacar, en este sentido, el abanico de temáticas que fueron objeto de atención por

\footnotetext{
${ }^{3}$ Desde sus primeros escarceos en el mundo de la antropología, Esteva consideró que ambos aspectos eran centrales en el discurso antropológico; así, ya en su período de formación en México adquirió: “[...] una fuerte conciencia de que sin esta interdisciplinariedad institucional plenamente integrada en una concepción integral, no es posible construir una antropología" (Esteva 1982: 7).
} 
parte de la publicación, temáticas que, en muchos casos, rompieron esquemas y abrieron fronteras al conocimiento y a la reflexión antropológica (ver gráfico n. $\left.{ }^{0} 1\right)^{4}$. De esta manera, debe hacerse constar cómo se abordaron ciertas problemáticas de forma novedosa, por ejemplo los problemas derivados de la relación entre inmigración, etnicidad y aculturación o los referidos a la transformación del mundo rural.

GRÁFICO 1.-MATERIAS

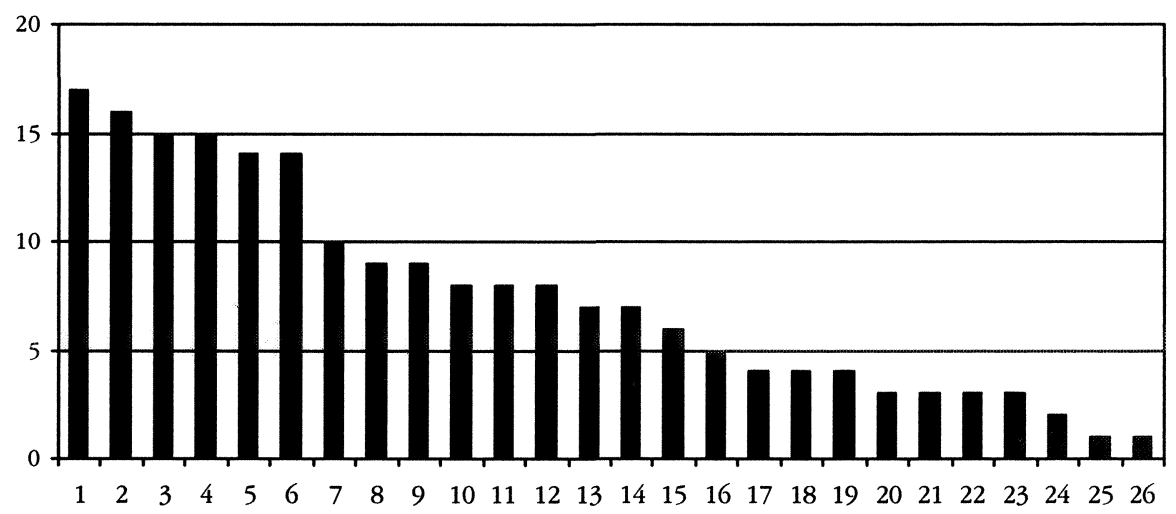

1 = Teoría antropológica (17)

2 = Historia de la Antropología (16)

$3=$ Organización social (15)

$4=$ Literatura y tradición oral (15)

5 = Parentesco, herencia, familia (14)

6 = Etnolingüística, Bilingüismo, Lingüística Semántica (14)

$7=$ Antropología Biológica (10)

8 = Cambio social (9)

$9=$ Etnohistoria (9)

10 = Antropología en España (8)

$11=$ Religión (8)

12 = Inmigración, aculturación (8)

13 = Campesinado (7)

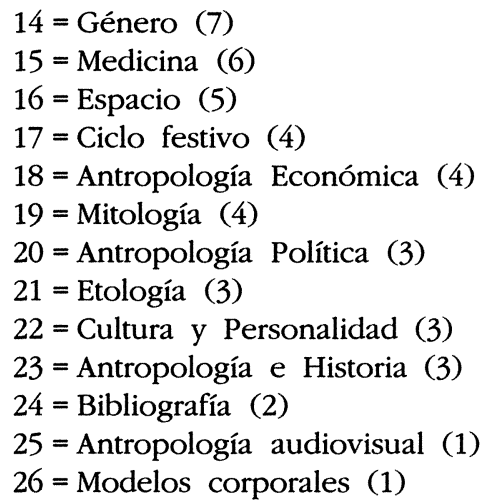

El segundo elemento a destacar es el notable papel que la teoría y el análisis histórico de la disciplina tuvieron en Ethnica, lo que significó una aportación considerable a la paulatina definición de la ciencia antropológica en España. En este sentido, es interesante recordar, a pesar de que no

${ }^{4}$ Los datos de los gráficos son estimativos; en la adscripción a una temática o área geográfica determinada se ha destacado el aspecto más distintivo de los trabajos. 
tengo datos concluyentes, el número de veces que determinados artículos de la publicación han sido, y son, citados.

La apertura temática se vio reflejada en el amplio abanico de autores que colaboraron en la publicación, ya fuesen consagrados (Bosch i Gimpera, Mead, Zamora, Alcina, Valls, Esteva, etc.) o noveles por aquel entonces (Buxó, Prat, Contreras, Frigolé, Terradas, Moreno y otros). Esta combinación dotó de una fuerte carga de credibilidad a la publicación. Si a ello se le añade la diversidad geográfica (vid. gráficos 2,3 y 4) de los temas tratados, se puede comprender mejor la especial significación que Ethnica tiene en la historia de la reciente antropología española.

La mencionada apertura y credibilidad se vieron ratificadas por el hecho de que una buena parte de los temas publicados fueron fruto del trabajo de campo, lo cual contribuyó a definir mucho mejor el objeto y la forma de la novel antropología institucionalizada española, que, en su proceso de instalación en el medio académico y social, no estuvo exenta de problemas. En este sentido, hay que recordar que Ethnica también sufrió las tensiones del momento, ya fuesen de orden económico (la falta de financiación hizo que a partir del volumen 12 -1976 - la revista pasase a ser anual en lugar de semestral), o de orden disciplinar (a partir de 1978 se aprecia una creciente participación de autores extranjeros y una sensible merma de colaboradores de la primera época).

Ethnica. Revista de Antropología definió una época de la antropología española, convirtiéndose, de una parte, en un símbolo de la institucionalización de la disciplina, con todas las esperanzas, alegrías y frustraciones que existen en todo proceso similar, y, de otra, en un referente de una concepción altamente holística e interdisciplinar de la antropología, concepción que el devenir ha ido difuminando, aunque la creciente caída de barreras teóricas y metodológicas apunta hacia un cambio de tendencia y hacia la recuperación de un tono similar al que Ethnica propugnó y divulgó.

GRÁFICO 2.-NÚMERO DE ARTíCULOS ESPAÑA/EXTERIOR

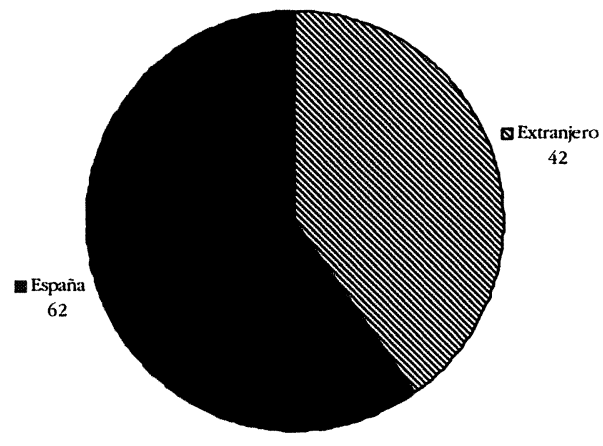


GRÁFICO 3.-DistribUCIÓN ARTíCULOS/ESPAÑA

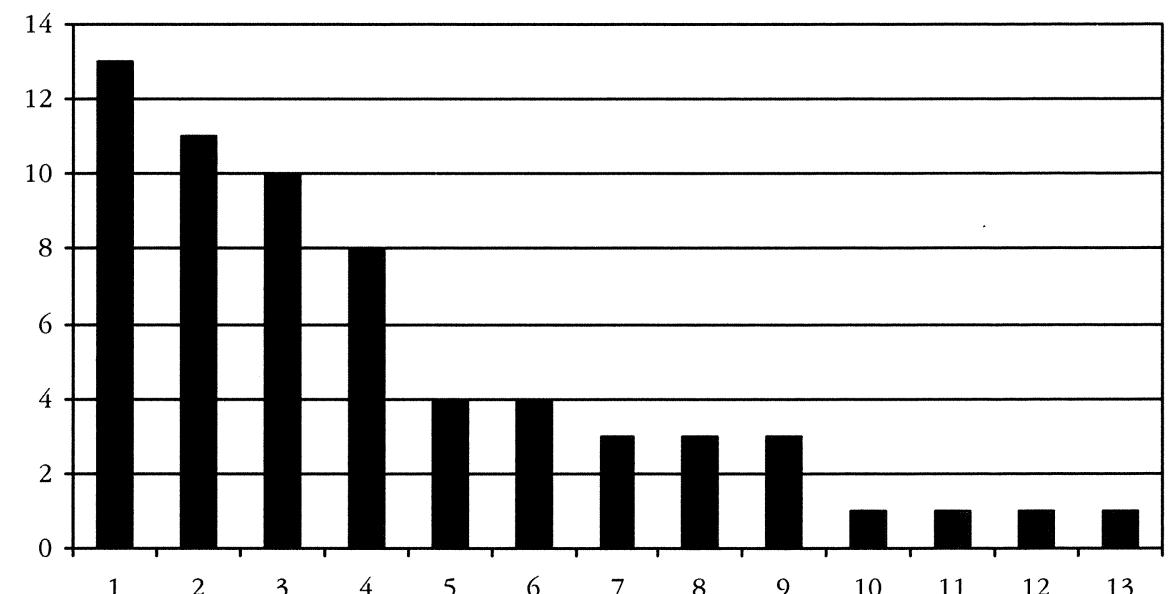

1 = Cataluña (13)

2 = Andalucía (11)

$3=$ España (general) (10)

4 = País Vasco (6)

$5=$ Pirineos $(4)$

6 = Castilla-León (4)

$7=$ Aragón (3)

$8=$ Galicia (3)

9= Castilla-La Mancha (3)

$10=$ Murcia $(1)$

$11=$ Canarias $(1)$

$12=$ Valencia $(1)$

$13=$ Norte de España (general) (1)

GRÁFICO 4.-EXTRANJERO: ÁREAS/ARTíCULOS

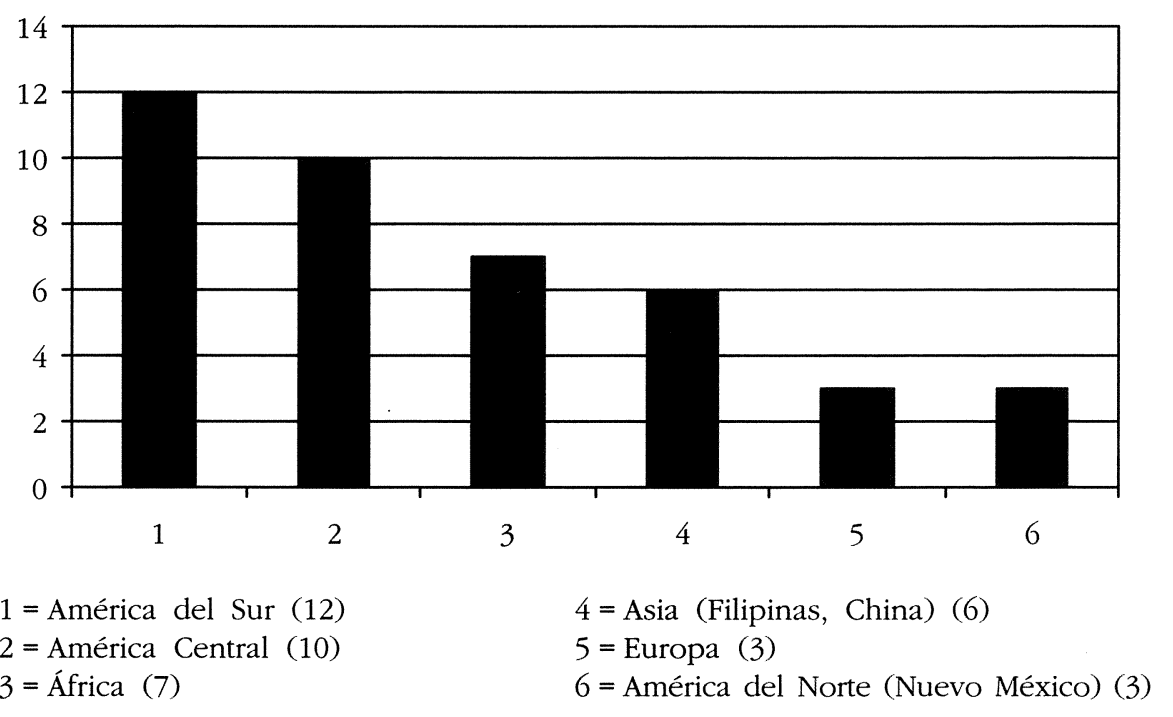




\section{BIBLIOGRAFÍA CITADA}

Aguirre, Á. 1986. "Claudio Esteva-Fabregat y la Etnología", en A. Aguirre (ed.), La Antropología Cultural en España: 397-455. Barcelona: PPU.

- 1994. "Esteva-Fabregat, Claudio", en Carmen Ortiz y Luis Ángel Sánchez (eds.), Diccionario histórico de la Antropología Española: 274-280. Madrid: CSIC.

AlCAÑız, B. 1994. "Ethnica. Revista de Antropologia", en Carmen Ortiz y Luis Ángel Sánchez (eds.), Diccionario bistórico de la Antropología Española: 281-283. Madrid: CSIC.

Buxó, M. J. 1994. "La confusión de los discursos: voces etnográficas y escrituras étnicas", en Ricardo Sanmartín (coord.), Antropología sin fronteras. Ensayos en honor de Carmelo Lisón: 153-167. Madrid: CIS.

Calvo, L. 1985. Claudio Esteva-Fabregat y la Antropología Aplicada, tesis de licenciatura, Universidad de Barcelona, inédita.

- 1993. "Claudio Esteva-Fabregat y la Antropología Española". Anuario de Historia de la Antropología Española 2: 27-34.

DELGADO, M. 1993. "Antropologia i nihilisme o qué és I'antropologia postmoderna". Revista d'Etnologia de Catalunya 3: 94-111.

EstevA, C. 1969. La Etnología española y sus problemas: Zaragoza: Institución Fernando el Católico.

- 1982. Autobiografia intelectual. Barcelona: Anthropos.

- 1993. "Antropología Cultural", en Ángel Aguirre (ed.), Diccionario temático de Antropologia: 25-32. Barcelona: Boixareu.

- 1994. "Antropologia: per a qué?", en Joan Vilà Valentí (coord.), Les relacions entre ciència $i$ societat a Catalunya, a la fi del segle XX: 161-223. Barcelona: Fundació Catalana per a la Recerca.

GEARY, C. M. 1990. "Impressions of the Africa Past: Interpreting Ethnographic Photographs from Cameroon". Visual Antbropology 3: 289-315.

GÓMEZ, E. 1990. "Sociología en España", en Salvador Giner y Luis Moreno (comps.), Sociología en España: 15-50. Madrid: CSIC.

PRAT, J. 1992. Antropología y Etnología. Madrid: Editorial Complutense y Caja de Ahorros y Monte de Piedad de Madrid.

SANMARTín, R. (coord.). 1994. Antropología sin fronteras. Ensayos en bonor de Carmelo Lisón. Madrid: CIS.

VOGET, F. W. 1984. Storia dell'etnologia contemporanea. Roma-Bari: Laeterza. 